\title{
On the Existence and Regularity of Hypersurfaces of Prescribed Gauss Curvature with Boundary
}

\section{Bo GUAN}

\begin{abstract}
In this paper we study the Dirichlet problem for some Monge-Ampère type equations on $\mathbb{S}^{n}$, which naturally arise in some geometric problems. The result then is applied to prove the existence of hypersurfaces in $\mathbb{R}^{n+1}$ of prescribed Gauss-Kronecker curvature and with fixed boundary.
\end{abstract}

1. Introduction. Let $\Omega$ be a smooth domain on the unit sphere $\mathbb{S}^{n} \subset$ $\mathbb{R}^{n+1}$, the Euclidean $(n+1)$-space. In this paper we study the classical solvability of the Dirichlet problem for the following equation of Monge-Ampère type

$$
\left(g^{-1} \operatorname{det}\left(u g_{i j}+\nabla_{i j} u\right)\right)^{1 / n}=\psi(x, u, \nabla u) \quad \text { in } \Omega,
$$

with boundary data

$$
u=\varphi \quad \text { on } \partial \Omega,
$$

where $g_{i j}$ denotes the metric of $\mathbb{S}^{n}, g=\operatorname{det}\left(g_{i j}\right)>0$ and $\varphi \in C^{\infty}(\partial \Omega), \psi>0$ is $C^{\infty}$ with respect to $(x, z, p) \in \bar{\Omega} \times \mathbb{R} \times T_{x} \mathbb{S}^{n}, T_{x} \mathbb{S}^{n}$ denotes the tangent space at $x \in \mathbb{S}^{n}$.

Equation (1.1) arises in various geometric problems related to the Gauss curvature such as the Minkowski problem (see Cheng and Yau [3] and the references therein). The Dirichlet problem was studied by Guan and Spruck [5] in connection with the problem of finding hypersurfaces in $\mathbb{R}^{n+1}$ of constant GaussKronecker curvature ( $K$-hypersurfaces). Let $M$ be a hypersurface in $\mathbb{R}^{n+1}$ which can be represented as a radial graph over $\Omega \subset \mathbb{S}^{n}$

$$
X(x)=\rho(x) x, \quad \rho>0, \quad x \in \bar{\Omega}
$$


where $X$ denotes the position vector of $M$ in $\mathbb{R}^{n+1}$. Then the function $u \equiv 1 / \rho$ solves (1.1) with

$$
\psi=K^{1 / n}\left(1+\left|\frac{\nabla u}{u}\right|^{2}\right)^{(n+2) /(2 n)}
$$

where $K$ denotes the Gauss curvature of $M$ (see [5]). When $K$ is a positive constant, the solvability of (1.1)-(1.2), with $\psi$ given by (1.4), is proved in [5] under some additional assumptions ((1.7) and (1.8) below). In the present paper we first extend this result to more general $\psi$ satisfying the assumption

$$
\psi(x, z, p) \text { is a convex function with respect to } p \in T_{x} \mathbb{S}^{n} \text {. }
$$

Note that this condition is satisfied by the function in (1.4).

We seek solutions $u$ for which equation (1.1) is elliptic. This is equivalent to the condition that

$$
\left\{u g_{i j}+\nabla_{i j} u\right\} \text { is positive definite. }
$$

A function $u \in C^{2}(\Omega)$ is called admissible with respect to (1.1) if it satisfies (1.6). Assume that

$$
\Omega \text { does not contain any hemisphere, }
$$

and that there exists an admissible strict subsolution $\underline{u} \in C^{\infty}(\bar{\Omega})$ to $(1.1)-(1.2)$, that is,

$$
\left(g^{-1} \operatorname{det}\left(\underline{u} g_{i j}+\nabla_{i j} \underline{u}\right)\right)^{1 / n} \geq \psi(x, \underline{u}, \nabla \underline{u})+\delta_{0} \quad \text { in } \quad \bar{\Omega},
$$

for some $\delta_{0}>0$ and $\underline{u}=\varphi$ on $\partial \Omega$. Define

$$
\mathcal{A}=\left\{u \in C^{\infty}(\bar{\Omega}):\left\{u g_{i j}+\nabla_{i j} u\right\}>0, u \geq \underline{u} \text { in } \bar{\Omega} \text { and } u=\underline{u} \text { on } \partial \Omega\right\} .
$$

We shall prove the following result:

Theorem 1.1. Under conditions (1.5), (1.7), and (1.8), there exists an admissible solution of $(1.1)-(1.2)$ in $C^{\infty}(\bar{\Omega})$. Moreover, any solution $u \in \mathcal{A}$ of (1.1)-(1.2) satisfies the a priori estimate

$$
\|u\|_{C^{2, \alpha}(\bar{\Omega})} \leq C=C\left(n, \Omega, \psi, \varphi, \underline{u}, \delta_{0}\right)
$$

We remark that the estimates in (1.9) were already proved in [5] for $\underline{u}>0$. However, since the linearized operator of $\operatorname{det}\left(u g_{i j}+\nabla_{i j} u\right)$ may have a nontrivial kernel, these estimates do not seem to yield directly the desired existence result. The monotone iteration approach in [5] also fails here for general $\psi$ because 
it makes use of the special property $\partial \psi / \partial u \leq 0$ of the function $\psi$ given by (1.4) with $K$ being a constant. Therefore we have to rederive (1.9) for a wider class of equations in order to apply degree theory to the proof of existence in Theorem 1.1.

An interesting question is whether Theorem 1.1 still holds if $\underline{u}$ is merely assumed to be a subsolution (i.e., $\delta_{0}=0$ in (1.8)). Based on the interior estimates for second derivatives we shall derive, we are able to prove

Theorem 1.2. Under conditions (1.5), (1.7), and (1.8) with $\delta_{0}=0$, Problem (1.1)-(1.2) admits a solution belonging to $C^{\infty}(\Omega) \cap C^{0,1}(\bar{\Omega})$.

As we observed, Problem (1.1)-(1.2) is closely related to the boundary value problem for hypersurfaces with prescribed Gauss-Kronecker curvature, which may be stated as follows. Given a disjoint collection

$$
\Gamma=\left\{\Gamma_{1}, \ldots, \Gamma_{m}\right\}
$$

of closed smooth embedded $(n-1)$ dimensional submanifolds of $\mathbb{R}^{n+1}$, and a positive smooth function $K$ defined in $\mathbb{R}^{n+1}$, decide whether there exist smooth hypersurfaces $M$ in $\mathbb{R}^{n+1}$ with Gauss curvature

$$
K[M](X)=K(X) \quad \text { for } X \in M, \quad \text { and boundary } \partial M=\Gamma .
$$

Locally this problem reduces to questions concerning Monge-Ampère type equations and we seek solutions for which the resulting equations are elliptic. This turns out to confine ourselves to strictly locally convex hypersurfaces (i.e. those whose principal curvatures are all positive; such a hypersurface locally lies on one side of its tangent plane at any point). Namely we will look for solutions of (1.11) among strictly locally convex hypersurfaces. There exists an elementary necessary condition for $\Gamma$ to bound such hypersurfaces: the second fundamental form of every $\Gamma_{i}$, as a submanifold of $\mathbb{R}^{n+1}$, must be non-degenerate everywhere. In particular, if some $\Gamma_{i}$ is contained in a hyperplane, it must be strictly convex. However, this condition is in general not sufficient; there are topological obstructions as shown by Rosenberg [8].

While the general solvability of Problem (1.11) still remains open, some important special cases have been investigated using the theory of Monge-Ampère equations. In the non-parametric setting, one is led to the Dirichlet problem for the equation of prescribed Gauss curvature in domains $\Omega \subset \mathbb{R}^{n}$

$$
\operatorname{det}\left(u_{i j}\right)=K(x, u)\left(1+|D u|^{2}\right)^{(n+2) / 2} \quad \text { in } \Omega,\left.\quad u\right|_{\partial \Omega}=\varphi .
$$

If $u$ is a solution of (1.12) then the Gauss curvature of $\operatorname{graph}(u)$ is given by $K$. By an existence theorem due to Caffarelli, Nirenberg and Spruck [1] and Krylov [7], (1.12) admits a strictly convex solution in $C^{\infty}(\bar{\Omega})$ when $\Omega$ is a strictly convex smooth domain and if there is a strictly convex subsolution in $C^{\infty}(\bar{\Omega})$ taking 
the same boundary value. For non-convex domains, Guan and Spruck [5], who extended the result of [6], proved the existence of a strictly locally convex solution in $C^{\infty}(\bar{\Omega})$ under the condition that there exists a strictly locally convex strict subsolution in $C^{\infty}(\bar{\Omega})$. Using an argument similar to the proof of Theorem 1.2, we shall prove the following result:

Theorem 1.3. Let $\Omega$ be a smooth bounded domain in $\mathbb{R}^{n}, K \in C^{\infty}(\bar{\Omega} \times$ $\mathbb{R}), K>0$ and $\varphi \in C^{\infty}(\partial \Omega)$. Suppose there exists a strictly locally convex subsolution $\underline{u} \in C^{\infty}(\bar{\Omega})$ to (1.12), i.e. $\underline{u}$ satisfies

$$
\operatorname{det}\left(\underline{u}_{i j}\right) \geq K(x, \underline{u})\left(1+|D \underline{u}|^{2}\right)^{(n+2) / 2} \quad \text { in } \Omega,\left.\quad \underline{u}\right|_{\partial \Omega}=\varphi .
$$

Then (1.12) admits a strictly locally convex solution $u \in C^{\infty}(\Omega) \cap C^{0,1}(\bar{\Omega})$ with $u \geq \underline{u}$ in $\bar{\Omega}$.

We should remark here that it is not known to us whether this solution has better regularity up to boundary. On the other hand, as we shall see Theorem 1.3 holds for some more general equations of Monge-Ampère type. Note that the novelty here, as in [5], lies in the fact that no geometric assumptions on $\partial \Omega$ are made.

Following the approach of Guan and Spruck [5] to the boundary value problem for K-hypersurfaces, in this paper we are mainly interested in seeking solutions to (1.11) which can be represented as radial graphs of form (1.3).

From Theorems 1.1 and 1.2 we obtain the following existence result which extends the main theorem of [5].

Theorem 1.4. Assume $\Gamma$ can be represented as a radial graph over the boundary of a smooth domain $\Omega$ on $\mathbb{S}^{n}$ (with a suitable choice of the center in $\mathbb{R}^{n+1}$ ) which does not contain any hemisphere. Assume there exists a strictly locally convex radial graph $\bar{X}(x)=\bar{\rho}(x) x$ over $\Omega$ with $\partial \bar{X}=\Gamma$ and Gauss curvature $\geq K(\bar{X}(x))+\delta_{0}$ for some constant $\delta_{0} \geq 0$. Then there exists a strictly locally convex hypersurface $M$ in $\mathbb{R}^{n+1}$ solving (1.11). Moreover, $M$ is a radial graph over $\Omega$ of form (1.3) with $\rho \in C^{\infty}(\bar{\Omega})$ if $\delta_{0}>0$ and $\rho \in C^{\infty}(\Omega) \cap C^{0,1}(\bar{\Omega})$ if $\delta_{0}=0$.

In particular, the $\delta_{0}=0$ case gives the following complement to Corollary 0.2 in $[5]$.

Corollary 1.5. Let $M$ be a closed smooth strictly convex hypersurface and $\Gamma=\partial D$ for a smooth subdomain $D \subset M$. Then $\Gamma$ bounds a smooth embedded hypersurface of constant Gauss curvature $K \equiv \inf _{X \in D} K[M](X)$.

This article is organized as follows: In Section 2 we establish a priori estimates up to the second derivatives for solutions of a family of equations of MongeAmpère type. The $C^{2, \alpha}$ and higher order estimates then follow from the work of Krylov [7] (see also Caffarelli et al. [2]) and the classical elliptic theory. These 
estimates are used to carry out the proof of Theorem 1.1 through a degree theory argument in Section 3. Theorem 1.2 and Theorem 1.3 are proved in Section 4, where we derive some useful interior estimates for second derivatives.

We conclude this introduction by recording some formulae for commuting covariant derivatives on $\mathbb{S}^{n}$. Throughout the paper, $\nabla$ denotes the covariant differentiation on $\mathbb{S}^{n}$. Let $e_{1}, \ldots, e_{n}$ be a local frame on $\mathbb{S}^{n}$. We use the notation $\nabla_{i}=\nabla_{e_{i}}, \nabla_{i j}=\nabla_{i} \nabla_{j}$, etc. For a differentiable function $v$ defined on $\mathbb{S}^{n}, \nabla v$ denotes the gradient, and $\nabla^{2} v$ the Hessian which is given by

$$
\nabla_{i j} v=\nabla_{i}\left(\nabla_{j} v\right)-\left(\nabla_{i} e_{j}\right) v
$$

We recall that $\nabla_{i j} v=\nabla_{j i} v$ and

$$
\begin{gathered}
\nabla_{i j k} v-\nabla_{j i k} v=g_{i k} \nabla_{j} v-g_{k j} \nabla_{i} v \\
\nabla_{i j k l} v-\nabla_{k l i j} v=2 g_{i j} \nabla_{k l} v-2 g_{k l} \nabla_{i j} v+g_{k j} \nabla_{i l} v-g_{i l} \nabla_{k j} v .
\end{gathered}
$$

2. A priori estimates. In this section we shall derive the estimate (1.9) for more general equations as follows. Let $h \in C^{2, \alpha}(\bar{\Omega})$ satisfy $h \geq \underline{u}$ in $\Omega$ and $h=\underline{u}$ on $\partial \Omega$. For $0 \leq t \leq 1$, we write $t^{\prime}=1-t$ and consider the equation

$$
F[u] \equiv\left(g^{-1} \operatorname{det}\left(\left(t u+t^{\prime} h\right) g_{i j}+\nabla_{i j} u\right)\right)^{1 / n}=\psi(x, u, \nabla u) \quad \text { in } \quad \bar{\Omega} .
$$

Theorem 2.1. Assume (1.5), (1.7), and (1.8) hold. Let $u \in C^{4, \alpha}(\bar{\Omega})$ be a solution of the Dirichlet problem (2.1)-(1.2) for some fixed $t \in[0,1]$ with

$$
\left\{\left(t u+t^{\prime} h\right) g_{i j}+\nabla_{i j} u\right\}>0 \quad \text { and } u \geq \underline{u} \text { in } \bar{\Omega} .
$$

Then

$$
\|u\|_{C^{2, \alpha}(\bar{\Omega})} \leq C, \quad \text { independent of } t,
$$

where $C$ depends on $\|h\|_{C^{2, \alpha}(\bar{\Omega})}$, in addition to the known data as in Theorem 1.1.

Proof. First we note that $\left(u+\max _{\Omega}|h|\right)$ is an admissible function. Thus it follows from the $C^{1}$ estimates in [5] that

$$
\|u\|_{C^{1}(\bar{\Omega})} \leq C_{0}, \quad \text { independent of } t .
$$

We remark that (2.4) holds for all $C^{2}$ functions satisfying (2.2) and $C_{0}$ does not depend on the function $\psi$. 
Turning to the second derivatives, we shall first assume a boundary bound

$$
\left|\nabla^{2} u\right| \leq C_{1} \quad \text { on } \partial \Omega
$$

and derive the global estimate

$$
\left|\nabla^{2} u\right| \leq C_{2} \quad \text { in } \bar{\Omega} .
$$

Then we come back to establish (2.5).

(a) Bounds for $\left|\nabla^{2} u\right|$ on $\bar{\Omega}$. Set

$$
M=\max _{x \in \bar{\Omega}} \max _{|\xi|=1, \xi \in T_{x} \mathbb{S}^{n}}\left(t u+t^{\prime} h+\nabla_{\xi}^{2} u\right) \exp \left\{\frac{a}{2}\left(u^{2}+|\nabla u|^{2}\right)-b t^{\prime}(u-\underline{u})\right\},
$$

where $a, b$ are positive constants to be determined later. In order to obtain (2.6) it suffices to derive a bound for $M$.

If the maximum $M$ occurs on $\partial \Omega$, then $M$ is estimated via the known estimates for $\|u\|_{C^{1}}$ and (2.5), and (2.6) thus follows. So assume $M$ is achieved at a point $x_{0} \in \Omega$ and for some unit vector $\xi \in T_{x_{0}} \mathbb{S}^{n}$. We may choose a smooth orthonormal local frame $e_{1}, \ldots, e_{n}$ about $x_{0}$ such that $e_{1}\left(x_{0}\right)=\xi$ and $\left\{\nabla_{i j} u\left(x_{0}\right)\right\}$ is diagonal. Set

$$
\lambda_{i}=\left(t u+t^{\prime} h+\nabla_{i i} u\right)\left(x_{0}\right)>0 \quad \text { for } i=1, \ldots, n .
$$

We need only estimate $\lambda_{1}$ from above.

The function $\log \left(t u+t^{\prime} h+\nabla_{11} u\right)+\frac{a}{2}\left(u^{2}+|\nabla u|^{2}\right)-b t^{\prime}(u-\underline{u})$ (defined near $\left.x_{0}\right)$ then attains a maximum at $x_{0}$. Thus at $x_{0}$, since $\nabla_{i j} u$ is diagonal,

(2.7) $\frac{\nabla_{i} u+\nabla_{i 11} u-t^{\prime} \nabla_{i}(u-h)}{\lambda_{1}}+a \nabla_{i} u\left(u+\nabla_{i i} u\right)-b t^{\prime} \nabla_{i}(u-\underline{u})=0$ for all $i$,

and

$$
\begin{aligned}
0 \geq \frac{\nabla_{i i} u+\nabla_{i i 11} u-t^{\prime} \nabla_{i i}(u-h)}{\lambda_{1}} & \\
- & \frac{\left(\nabla_{i} u+\nabla_{i 11} u-t^{\prime} \nabla_{i}(u-h)\right)^{2}}{\lambda_{1}^{2}}+a \nabla_{i i} u\left(u+\nabla_{i i} u\right) \\
& +a \sum_{j} \nabla_{j} u\left(\nabla_{i} u \delta_{i j}+\nabla_{i i j} u\right)-b t^{\prime} \nabla_{i i}(u-\underline{u}),
\end{aligned}
$$

for all $i$. 
Rewrite Equation (2.1) as

$$
\log \operatorname{det}\left(u \delta_{i j}+\nabla_{i j} u-t^{\prime}(u-h) \delta_{i j}\right)=n \log \psi
$$

and differentiate it twice to obtain at $x_{0}$,

$$
\begin{gathered}
\sum_{i} \frac{\nabla_{j} u+\nabla_{j i i} u-t^{\prime} \nabla_{j}(u-h)}{\lambda_{i}}=n \nabla_{j}(\log \psi) \text { for all } j, \\
\sum_{i} \frac{\nabla_{11} u+\nabla_{11 i i} u-t^{\prime} \nabla_{11}(u-h)}{\lambda_{i}} \\
\quad-\sum_{i, j} \frac{\left(\nabla_{1} u \delta_{i j}+\nabla_{1 i j} u-t^{\prime} \nabla_{1}(u-h) \delta_{i j}\right)^{2}}{\lambda_{i} \lambda_{j}}=n \nabla_{11}(\log \psi) .
\end{gathered}
$$

Using (1.13), we write for $i \geq 2$,

$$
\begin{aligned}
\left(\nabla_{i} u+\nabla_{i 11} u-t^{\prime} \nabla_{i}(u-h)\right)^{2}= & \left(\nabla_{1} u \delta_{1 i}+\nabla_{11 i} u-t^{\prime} \nabla_{1}(u-h) \delta_{1 i}\right)^{2} \\
& -2 t^{\prime} \nabla_{i}(u-h)\left(\nabla_{i} u+\nabla_{i 11} u-t^{\prime} \nabla_{i}(u-h)\right) \\
& -\left(t^{\prime}\right)^{2}\left(\nabla_{i}(u-h)\right)^{2}
\end{aligned}
$$

and combine it with (2.8) to derive

$$
\begin{aligned}
& \sum_{i, j} \frac{\left(\nabla_{1} u \delta_{i j}+\nabla_{1 i j} u-t^{\prime} \nabla_{1}(u-h) \delta_{i j}\right)^{2}}{\lambda_{i} \lambda_{j}}-\sum_{i} \frac{\left(\nabla_{i} u+\nabla_{i 11} u-t^{\prime} \nabla_{i}(u-h)\right)^{2}}{\lambda_{1} \lambda_{i}} \\
\geq & -C a-C(a+b) t^{\prime} \sum_{i} \frac{1}{\lambda_{i}} .
\end{aligned}
$$

Multiplying (2.8) by $\lambda_{1} \lambda_{i}^{-1}$, from (2.10) and (1.14), we find with $f=n \log \psi$,

$$
\begin{aligned}
0 \geq(1+ & \left.t^{\prime}\right) \sum_{i} \frac{1}{\lambda_{i}}\left(\nabla_{11} u-\nabla_{i i} u\right) \\
& -t^{\prime} \sum_{i} \frac{1}{\lambda_{i}}\left(\nabla_{11} h-\nabla_{i i} h\right)+\nabla_{11} f-C a-C(a+b) t^{\prime} \sum_{i} \frac{1}{\lambda_{i}} \\
& +a \lambda_{1} \sum_{i} \frac{1}{\lambda_{i}} \nabla_{i i} u\left(u+\nabla_{i i} u\right)+a \lambda_{1} \sum_{i, j} \frac{1}{\lambda_{i}} \nabla_{j} u\left(\nabla_{i} u \delta_{i j}+\nabla_{i i j} u\right) \\
& -b \lambda_{1} t^{\prime} \sum_{i} \frac{\nabla_{i i}(u-\underline{u})}{\lambda_{i}} .
\end{aligned}
$$


The first two terms on the right-hand side are bounded below by $-C\left(1+t^{\prime} \sum \lambda_{i}^{-1}\right)$. Since $\left\{\underline{u} \delta_{i j}+\nabla_{i j} \underline{u}\right\}$ is positive definite, we have

$$
\sum_{i} \frac{\nabla_{i i}(u-\underline{u})}{\lambda_{i}} \leq \sum_{i} \frac{\lambda_{i}-\left(\underline{u}+\nabla_{i i} \underline{u}\right)}{\lambda_{i}} \leq n-c_{1} \sum_{i} \frac{1}{\lambda_{i}}
$$

for some $c_{1}>0$. We calculate

$$
\begin{aligned}
\nabla_{11} f & \geq \sum_{j} f_{p_{j}} \nabla_{11 j} u-C\left(1+\lambda_{1}^{2}\right) \\
& \geq-a \lambda_{1} \sum_{j} f_{p_{j}} \nabla_{j} u \nabla_{j j} u-C\left(1+(a+b) \lambda_{1}+\lambda_{1}^{2}\right)
\end{aligned}
$$

by (2.8). From (2.9) and (1.13),

$$
\begin{aligned}
\sum_{i, j} \frac{1}{\lambda_{i}} \nabla_{j} u\left(\nabla_{i} u \delta_{i j}+\nabla_{i i j} u\right) & =\sum_{j} \nabla_{j} u \nabla_{j} f+t^{\prime} \sum_{i, j} \frac{1}{\lambda_{i}} \nabla_{j} u \nabla_{j}(u-h) \\
& \geq \sum_{j} f_{p_{j}} \nabla_{j} u \nabla_{j j} u-C-C t^{\prime} \sum_{i} \frac{1}{\lambda_{i}} .
\end{aligned}
$$

Thus,

$$
\begin{array}{r}
\nabla_{11} f+a \lambda_{1} \sum_{i, j} \frac{1}{\lambda_{i}} \nabla_{j} u\left(\nabla_{i i j} u+\nabla_{i} u \delta_{i j}\right) \\
\geq \quad-C-C \lambda_{1}\left(a+b+\lambda_{1}\right)-C a \lambda_{1} t^{\prime} \sum_{i} \frac{1}{\lambda_{i}}
\end{array}
$$

and from (2.11),

$$
0 \geq(a-C) \lambda_{1}^{2}-C(a+b) \lambda_{1}-C+\left(\left(b c_{1}-C a\right) \lambda_{1}-C(1+a+b)\right) t^{\prime} \sum_{i} \frac{1}{\lambda_{i}}
$$

Consequently, we either have

$$
\begin{gathered}
(a-C) \lambda_{1}^{2}-C(a+b) \lambda_{1}-C \leq 0, \quad \text { or } \\
\left(b c_{1}-C a\right) \lambda_{1}-C(1+a+b) \leq 0 .
\end{gathered}
$$

Thus, we can choose $b \gg a \gg 1$ to obtain a desired upper bound for $\lambda_{1}$. 
(b) Bounds for $\left|\nabla^{2} u\right|$ on $\partial \Omega$. About a point $x_{0} \in \partial \Omega$, let $e_{1}, \ldots, e_{n}$ be a local orthonormal frame on $\mathbb{S}^{n}$ obtained by parallel translation of a local orthonormal frame on $\partial \Omega$ and the interior unit normal vector field to $\partial \Omega$ along the geodesics perpendicular to $\partial \Omega$ on $\mathbb{S}^{n}$. We assume $e_{n}$ is the parallel translation of the unit normal field on $\partial \Omega$ and set

$$
B_{\alpha \beta}=\left\langle\nabla_{\alpha} e_{\beta}, e_{n}\right\rangle, \quad 1 \leq \alpha, \beta \leq n-1
$$

On $\partial \Omega$ we have $u-\underline{u}=0$, so

$$
\nabla_{\alpha}(u-\underline{u})=0 \quad \text { for } \alpha<n,
$$

and

$$
\begin{aligned}
\nabla_{\alpha \beta}(u-\underline{u}) & =\nabla_{\alpha}\left(\nabla_{\beta}(u-\underline{u})\right)-\sum_{i}\left\langle\nabla_{\alpha} e_{\beta}, e_{i}\right\rangle \nabla_{i}(u-\underline{u}) \\
& =-B_{\alpha \beta} \nabla_{n}(u-\underline{u}) \text { for } \alpha, \beta<n .
\end{aligned}
$$

It follows that

$$
\left|\nabla_{\alpha \beta} u\left(x_{0}\right)\right| \leq C, \quad \alpha, \beta<n .
$$

We proceed to estimate the derivatives $\nabla_{n \alpha} u$ for $\alpha \leq n$. Let $L$ be the linearized operator (at $u$ ) of $F[u]$, that is

$$
L v=\sum_{i, j} F^{i j}\left(\nabla_{i j} v+t v \delta_{i j}\right), \quad \text { for } v \in C^{2}(\bar{\Omega}),
$$

where $\left\{F^{i j}\right\}$ is the inverse matrix of

$$
\left\{\frac{n}{F[u]}\left(\left(t u+t^{\prime} h\right) \delta_{i j}+\nabla_{i j} u\right)\right\}>0 .
$$

For fixed $\alpha \leq n$, we find using (1.13)

$$
\begin{aligned}
L \nabla_{\alpha} u & =\sum_{i, j} F^{i j}\left(t \nabla_{\alpha} u \delta_{i j}+\nabla_{i j \alpha} u\right) \\
& =\nabla_{\alpha}(F[u])+\nabla_{\alpha}\left(u-t^{\prime} h\right) \sum_{i} F^{i i}-\sum_{i} F^{i \alpha} \nabla_{i} u .
\end{aligned}
$$

We introduce another linear operator

$$
\mathcal{L}=L-\psi_{p_{i}}(x, u, \nabla u) \nabla_{i}-c,
$$


where

$$
c=\max _{x \in \bar{\Omega}} \max _{\underline{u} \leq z \leq u}\left|\psi_{z}(x, z, D \underline{u})\right|<\infty .
$$

From (2.14) we have for $\alpha \leq n$,

$$
\left|\mathcal{L} \nabla_{\alpha}(u-\underline{u})\right| \leq C\left(1+\sum_{i} F^{i i}\right)
$$

We want to construct barriers in a small neighborhood of $x_{0}$. Consider the distance function

$$
d(x) \equiv \operatorname{dist}_{\mathbb{S}^{n}}\left(x, x_{0}\right),
$$

and set

$$
\Omega_{\delta}=\{x \in \Omega: d(x)<\delta\} .
$$

Since $\nabla_{i j} d^{2}\left(x_{0}\right)=2 \delta_{i j}$, by choosing $\delta$ small enough, we may assume

$$
\left\{\delta_{i j}\right\} \leq\left\{\nabla_{i j} d^{2}\right\} \leq 2\left\{\delta_{i j}\right\} \quad \text { in } \Omega_{\delta} .
$$

Lemma 2.2. There is a uniform positive constant $\varepsilon_{1}$ independent of $t$ such that

$$
\mathcal{L}(\underline{u}-u) \geq \varepsilon_{1}\left(1+\sum F^{i i}\right) \quad \text { in } \quad \Omega_{\delta} .
$$

Proof. Consider the function $w=\underline{u}-\varepsilon d^{2}$. In view of (2.16), for $\varepsilon>0$ small enough,

$$
F[w] \geq F[\underline{u}]-\frac{\delta_{0}}{2} \geq \psi(x, \underline{u}, \nabla \underline{u})+\frac{\delta_{0}}{2} .
$$

By the concavity of $F$ and the convexity of $\psi(\cdot, \cdot, p)$ in $p$, we then find

$$
\begin{aligned}
L(w-u) & \geq F[w]-F[u] \\
& \geq \psi(x, \underline{u}, \nabla \underline{u})+\frac{\delta_{0}}{2}-\psi(x, u, \nabla u) \\
& \geq \sum_{i} \psi_{p_{i}}(x, u, \nabla u) \nabla_{i}(\underline{u}-u)+\psi_{z}(x, z, \nabla \underline{u})(\underline{u}-u)+\frac{\delta_{0}}{2},
\end{aligned}
$$

for some $z=z(x)$ with $\underline{u} \leq z \leq u$. The proof is thus complete. 
Using Lemma 2.2, we may employ a barrier function in $\Omega_{\delta}$ of the form

$$
v=A(u-\underline{u})+B d^{2},
$$

to estimate $\nabla_{\alpha n} u$. For the mixed normal tangential derivatives $\nabla_{\alpha n} u\left(x_{0}\right), \alpha<n$, we may choose $A \gg B \gg 1$ so that

$$
\mathcal{L}\left(v \pm \nabla_{\alpha}(u-\underline{u})\right) \leq 0 \quad \text { in } \Omega_{\delta},
$$

and

$$
v \pm \nabla_{\alpha}(u-\underline{u}) \geq 0 \quad \text { on } \partial \Omega_{\delta},
$$

since $\nabla_{\alpha}(u-\underline{u})=0$ on $\partial \Omega \cap \Omega_{\delta}$, and $\left|\nabla_{\alpha}(u-\underline{u})\right| \leq C$ in $\Omega$. We observe that for $\delta$ sufficiently small, the operator $L$ has a trivial kernel on $\Omega_{\delta}$. Thus we may apply the maximum principle to conclude

$$
v \geq\left|\nabla_{\alpha}(u-\underline{u})\right| \text { in } \Omega_{\delta} .
$$

Consequently,

$$
\left|\nabla_{n \alpha} u\left(x_{0}\right)\right| \leq \nabla_{n} v\left(x_{0}\right)+\left|\nabla_{n \alpha} \underline{u}\left(x_{0}\right)\right| \leq C, \quad \alpha<n .
$$

It remains to estimate the double normal derivative $\nabla_{n n} u$. Since $\Delta_{s} u+n u \geq$ 0 , it suffices to derive an upper bound

$$
\nabla_{n n} u \leq C \quad \text { on } \partial \Omega .
$$

The following proof is inspired by an idea of Trudinger [9] and simplifies our original argument. For $x \in \partial \Omega$ let

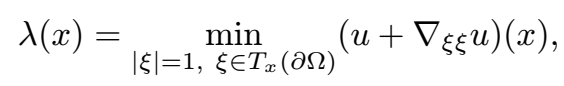

and assume that $\lambda(x)$ is minimized at $x_{0} \in \partial \Omega$ with $\xi=e_{1}\left(x_{0}\right)$, that is

$$
\left(u+\nabla_{11} u\right)\left(x_{0}\right) \leq\left(u+\nabla_{\xi \xi} u\right)(x)
$$

for all $x \in \partial \Omega$ and any unit vector $\xi \in T_{x}(\partial \Omega)$. As in [1], (2.20) will follow from Equation (2.1) if we can prove

$$
\lambda\left(x_{0}\right) \geq c_{0}>0
$$

for some uniform constant $c_{0}$. We consider two possible cases: 
Case 1. $\nabla_{11} u\left(x_{0}\right) \geq \frac{1}{2} \nabla_{11} \underline{u}\left(x_{0}\right)$. Then we are done since $\left(\underline{u}+\nabla_{11} \underline{u}\right)\left(x_{0}\right) \geq$ $c_{1}>0$ for some uniform $c_{1}>0$.

Case 2. $\nabla_{11} u\left(x_{0}\right)<\frac{1}{2} \nabla_{11} \underline{u}\left(x_{0}\right)$. From (2.12) we have

$$
\nabla_{11} u=\nabla_{11} \underline{u}-B_{11} \nabla_{n}(u-\underline{u}) \quad \text { on } \partial \Omega .
$$

It follows that

$$
B_{11}\left(x_{0}\right) \nabla_{n}(u-\underline{u})\left(x_{0}\right) \geq \frac{1}{2} \nabla_{11} \underline{u}\left(x_{0}\right) \geq \frac{c_{1}}{2}
$$

and, for $x \in \partial \Omega$,

$B_{11}(x) \nabla_{n}(u-\underline{u})(x) \leq\left(\underline{u}+\nabla_{11} \underline{u}\right)(x)-\left(\underline{u}+\nabla_{11} \underline{u}\right)\left(x_{0}\right)+B_{11}\left(x_{0}\right) \nabla_{n}(u-\underline{u})\left(x_{0}\right)$

since $\left.\left(u+\nabla_{11} u\right)\right|_{\partial \Omega}$ is minimized at $x_{0}$ and $u=\underline{u}$ on $\partial \Omega$. Note that since $B_{11}$ is smooth near $\partial \Omega$ and $0<\nabla_{n}(u-\underline{u}) \leq C$, we must have

$$
B_{11} \geq c_{2}>0 \quad \text { on } \Omega_{\delta}
$$

for some uniform $c_{2}>0$, if $\delta$ is chosen sufficiently small. Therefore,

$$
\nabla_{n}(u-\underline{u})(x) \leq \Psi(x) \text { for } x \in \Omega_{\delta} \cap \partial \Omega \text { and } \nabla_{n}(u-\underline{u})\left(x_{0}\right)=\Psi\left(x_{0}\right)
$$

where $\Psi(x)=B_{11}^{-1}(x)\left[\left(\underline{u}+\nabla_{11} \underline{u}\right)(x)-\left(\underline{u}+\nabla_{11} \underline{u}\right)\left(x_{0}\right)+B_{11}\left(x_{0}\right) \nabla_{n}(u-\underline{u})\left(x_{0}\right)\right]$.

We observe that $\Psi$ is smooth in $\Omega_{\delta}$. Thus by (2.15), (2.24) and Lemma 2.2, we may choose $A \gg B \gg 1$ such that

$$
\begin{gathered}
v+\Psi-\nabla_{n}(u-\underline{u}) \geq 0 \quad \text { on } \partial \Omega_{\delta}, \\
\mathcal{L}\left(v+\Psi-\nabla_{n}(u-\underline{u})\right) \leq 0 \quad \text { in } \Omega_{\delta} .
\end{gathered}
$$

where $v$ as in (2.18). As before, the maximum principle then yields

$$
v+\Psi-\nabla_{n}(u-\underline{u}) \geq 0 \quad \text { in } \Omega_{\delta} .
$$

Consequently, since $v+\Psi-\nabla_{n}(u-\underline{u})=0$ at $x_{0}$, we have

$$
\nabla_{n n} u\left(x_{0}\right) \leq C .
$$

We have shown that the eigenvalues of $\left\{u \delta_{i j}\left(x_{0}\right)+\nabla_{i j} u\left(x_{0}\right)\right\}$ are all bounded (they are also all positive). On the other hand, in virtue of Equation (2.1), the product of these eigenvalues are bounded below from zero by a uniform constant. 
Thus each of them must be bounded below from zero. In particular, we obtain the estimate in (2.21) which in turn implies (2.20). This completes the proof of (2.5).

Finally, as mentioned in the introduction, we may apply the results of Krylov [7] to obtain the $C^{2, \alpha}$ estimate (2.3).

3. Proof of Theorem 1.1. In this section we make use of the a priori estimates established in Section 2 and degree theory to prove the existence of solutions to (1.1)-(1.2) as in [1]. To begin with, let $u^{0} \in \mathcal{A}$ be a solution of

$$
\left(g^{-1} \operatorname{det}\left(u^{0} g_{i j}+\nabla_{i j} u^{0}\right)\right)^{1 / n}=\psi(x, \underline{u}, \nabla \underline{u}) \quad \text { in } \Omega,
$$

with $u^{0}=\varphi$ on $\partial \Omega$. It follows from [5] that such a solution $u^{0}$ exists and satisfies

$$
u^{0}>\underline{u} \quad \text { in } \Omega \text { and } \nabla_{\nu}\left(u^{0}-\underline{u}\right)>0 \text { on } \partial \Omega,
$$

where $\nabla_{\nu}$ is the interior normal differentiation on $\partial \Omega$.

For $0 \leq t \leq 1$, consider the Dirichlet problem for the equation

$$
F^{t}[u] \equiv\left(g^{-1} \operatorname{det}\left(\left(t u+t^{\prime} u^{0}\right) g_{i j}+\nabla_{i j} u\right)\right)^{1 / n}=\psi^{t}(x, u, \nabla u) \quad \text { in } \Omega,
$$

with boundary condition (1.2), where $t^{\prime}=1-t$ and

$$
\psi^{t}(x, u, \nabla u)=t \psi(x, u, \nabla u)+t^{\prime} \psi(x, \underline{u}, \nabla \underline{u}) .
$$

Let $\mathcal{A}^{t}$ denote the collection of admissible functions associated with (3.3):

$$
\mathcal{A}^{t}=\left\{u \in C^{\infty}(\bar{\Omega}):\left\{\left(t u+t^{\prime} u^{0}\right) g_{i j}+\nabla_{i j} u\right\}>0, u \geq \underline{u} \text { in } \bar{\Omega},\left.u\right|_{\partial \Omega}=\varphi\right\} .
$$

Note that for each $t \in[0,1], \underline{u} \in \mathcal{A}^{t}$ and is a strict subsolution of (3.3), and $\psi^{t}$ satisfies (1.5). By Theorem 2.1 and the elliptic regularity theory we thus have the $a$ priori estimate for each $k \geq 1$

$$
\|u\|_{C^{k}(\bar{\Omega})} \leq C, \text { independent of } t
$$

for all solutions $u \in \mathcal{A}^{t}$ of (3.3)-(1.2).

Consider the open convex cone of functions in $C_{0}^{5}(\bar{\Omega})$

$$
\mathcal{C}=\left\{v \in C_{0}^{5}(\bar{\Omega}): v>0 \text { in } \Omega \text { and } \nabla_{\nu} v>0 \text { on } \partial \Omega\right\} .
$$


Here $C_{0}^{5}(\bar{\Omega})$ is the space of functions in $C^{5}(\bar{\Omega})$ which vanish on $\partial \Omega$. We want to prove for each $t \in[0,1]$ there exists a solution of the form

$$
u=\underline{u}+v, \quad v \in \mathcal{C}
$$

to Problem (3.3)-(1.2). First we observe that there is no solution $u=\underline{u}+v \in \mathcal{A}^{t}$ of (3.3)-(1.2) with $v$ on the boundary of $\mathcal{C}$. For if $u \in \mathcal{A}^{t}$ is a solution of (3.3)(1.2), then

$F^{t}[u]-F^{t}[\underline{u}]-t(\psi(x, u, \nabla u)-\psi(x, \underline{u}, \nabla \underline{u}))=-\left(F^{t}[\underline{u}]-\psi(x, \underline{u}, \nabla \underline{u})\right) \leq-\delta_{0}<0$.

Since $u \geq \underline{u}$, the maximum principle is valid to conclude that $u-\underline{u} \in \mathcal{C}$.

According to (3.4) we can choose $r>0$ sufficiently large so that (3.3)-(1.2) does not have solution in $\mathcal{A}^{t}$ of the form (3.5) with $\|v\|_{C^{5}(\bar{\Omega})}=r$. Set

$$
\mathcal{C}_{r}=\left\{v \in \mathcal{C}:\|v\|_{C^{5}(\bar{\Omega})}<r\right\}
$$

and for each $v \in \overline{\mathcal{C}_{r}}$ and $t \in[0,1]$,

$\mathcal{A}^{t}[v]=\left\{u \in C^{\infty}(\bar{\Omega}):\left\{\left(t \underline{u}+t v+t^{\prime} u^{0}\right) g_{i j}+\nabla_{i j} u\right\}>0, u \geq \underline{u}\right.$ in $\left.\bar{\Omega},\left.u\right|_{\partial \Omega}=\varphi\right\}$.

From (2.2) and the remark following it, we have the a priori estimate

$$
\|u\|_{C^{1}(\bar{\Omega})} \leq C_{0}^{\prime} \quad \text { for all } u \in \mathcal{A}^{t}[v]
$$

where $C_{0}^{\prime}$ depends on $\|v\|_{C^{0}}$ but does not on $t$. It follows that

$$
\Lambda \equiv \sup _{0 \leq t \leq 1} \sup _{v \in \overline{\mathcal{C}_{r}}} \sup _{u \in \mathcal{A}^{t}[v]} \sup _{x \in \bar{\Omega}} \frac{\left|\psi_{z}(x, u, \nabla u)\right|}{\psi(x, u, \nabla u)}<\infty
$$

Now for $0 \leq t \leq 1$ and fixed $v \in \overline{\mathcal{C}}_{r}$, consider the equation

$$
\left(g^{-1} \operatorname{det}\left(\left(t \underline{u}+t v+t^{\prime} u^{0}\right) g_{i j}+\nabla_{i j} u\right)\right)^{1 / n}=\eta^{t}(x, u, \nabla u) \quad \text { in } \Omega,
$$

where $\eta^{t}(x, z, p)=\psi^{t}(x, z, p) e^{t \Lambda(z-\underline{u}-v)}$. We observe that $\eta^{t}$ is a convex function with respect to $p$ and $\underline{u}$ is a strict subsolution of (3.7). It thus follows from Theorem 2.1 that any solution $u \in \mathcal{A}^{t}[v]$ of (3.7)-(1.2) satisfies the a priori estimate

$$
\|u\|_{C^{2, \alpha}(\bar{\Omega})} \leq C, \quad \text { independent of } t \text { and } v .
$$

Since $\eta_{z}^{t}(x, u, \nabla u) \geq 0$ for any $u \in \mathcal{A}^{t}[v]$, the estimate above and the implicit function theorem (the method of continuity) imply the existence of a unique solution $u^{t} \in \mathcal{A}^{t}[v]$ to (3.7)-(1.2). For $t=0$, this solution is our $u^{0}$. 
From elliptic theory, the map

$$
T^{t} v=u^{t}-\underline{u}
$$

is compact in $C^{5}$. On the other hand, we have seen that there are no solutions of

$$
v-T^{t} v=0
$$

on the boundary of $\mathcal{C}_{r}$. Thus, the degree

$$
\operatorname{deg}\left(I-T^{t}, \mathcal{C}_{r}, 0\right)=\gamma
$$

is well defined and independent of $t$. For $t=0$, (3.9) has a solution $v^{0}=u^{0}-\underline{u} \in$ $\mathcal{C}_{r}$. By the maximum principle, the linearized operator of $\operatorname{det}\left(u^{0} g_{i j}+\nabla_{i j} u\right)$, linearized at $u^{0}$, is invertible. It follows that $v^{0}$ is a regular point of $I-T^{0}$. Consequently $\gamma= \pm 1$, and (3.9) has a solution for all $0 \leq t \leq 1$. The function $u^{1}=\underline{u}+v^{1}$ is then a solution of (1.1)-(1.2). The elliptic regularity theory implies that $\bar{u}^{1} \in C^{\infty}(\bar{\Omega})$.

This completes the proof of Theorem 1.1.

4. Interior estimates for second derivatives. In order to prove Theorem 1.2, we need the following interior estimate for the second derivatives of admissible solutions of (1.1)-(1.2).

Theorem 4.1. Let $u \in C^{\infty}(\bar{\Omega})$ be an admissible solution of (1.1)-(1.2). Assume that $\psi$ satisfies (1.5) and that there exists an admissible function $v \in$ $C^{2}(\bar{\Omega})$ satisfying $v \geq u$ in $\Omega, v=\varphi$ on $\partial \Omega$ and

(4.1) $\frac{\varepsilon_{0}}{2} \leq F[v] \equiv\left(g^{-1} \operatorname{det}\left(v g_{i j}+\nabla_{i j} v\right)\right)^{1 / n} \leq \psi(x, v, \nabla v)-\varepsilon_{0}, \quad$ in $\bar{\Omega}$, for some $\varepsilon_{0}>0$. Then

$$
\left|\nabla^{2} u(x)\right| \leq \frac{C}{\operatorname{dist}(x, \partial \Omega)}, \quad \text { for } x \in \Omega,
$$

for some uniform constant $C$ depending on $n, \Omega, \varepsilon_{0},\|u\|_{C^{1}(\bar{\Omega})}$, and $\|v\|_{C^{2}(\bar{\Omega})}$.

We first prove the following lemma.

Lemma 4.2. Let $u, v$ be as in Theorem 4.1 and assume that $\psi$ satisfies (1.5). Then

$$
(v-u)(x) \geq c_{0} \operatorname{dist}_{\mathbb{S}^{n}}(x, \partial \Omega), \quad x \in \Omega,
$$

for some uniform constant $c_{0}>0$. 
Proof. Given $y \in \Omega$, let $r=\frac{1}{3} \operatorname{dist}_{\mathbb{S}^{n}}(y, \partial \Omega)$ and let $h$ be the solution of

$$
\Delta_{s} h+n h=0 \text { in } B_{r}(y), \quad h=v \text { on } \partial B_{r}(y),
$$

where $\Delta_{s}$ is the Laplace-Beltrami operator on $\mathbb{S}^{n}$ and $B_{r}(y)=\left\{x \in \mathbb{S}^{n}\right.$ : $\left.\operatorname{dist}_{\mathbb{S} n}(x, y)<r\right\}$ is the geodesic ball of radius $r$ about $y$. Since $r \leq \frac{\pi}{3}$, the first eigenvalue of $B_{r}(y)$ is larger than $n$ and thus (4.4) has a unique solution. Moreover, since from (4.1),

$$
\Delta_{s} v+n v \geq \frac{n \varepsilon_{0}}{2}
$$

a standard barrier argument shows that

$$
(h-v)(x) \geq \theta_{0} \operatorname{dist}_{\mathbb{S}^{n}}\left(x, \partial B_{r}(y)\right) \text { for } x \in B_{r}(y)
$$

for some $\theta_{0}>0$.

For $t \geq 0$, consider the function $w^{t} \equiv v-t(h-v)$ in $B_{r}(y)$; from (4.1) we see that there exists some $t_{0}>0$ sufficiently small depending on $\varepsilon_{0}$ such that

$$
\left\{w^{t} g_{i j}+\nabla_{i j} w^{t}\right\}>0
$$

and

$$
F\left[w^{t}\right] \equiv\left(g^{-1}\left(\operatorname{det}\left(w^{t} g_{i j}+\nabla_{i j} w^{t}\right)\right)^{1 / n} \leq \psi\left(x, w^{t}, \nabla w^{t}\right)\right.
$$

for all $t \in\left[0, t_{0}\right]$. Let $L^{t}$ be the linearized operator of $F$ at $w^{t}$; as in the proof of Lemma 2.2 , we have, for all $t \in\left[0, t_{0}\right]$,

$$
\begin{aligned}
L^{t}\left(u-w^{t}\right) & \geq F[u]-F\left[w^{t}\right] \\
& \geq \psi(x, u, \nabla u)-\psi\left(x, w^{t}, \nabla w^{t}\right) \\
& \geq \psi_{p_{i}}\left(x, w^{t}, \nabla w^{t}\right) \nabla_{i}\left(u-w^{t}\right)+\psi_{z}\left(x, z, \nabla w^{t}\right)\left(u-w^{t}\right) .
\end{aligned}
$$

for some $z=z(x)$ between $u(x)$ and $w^{t}(x)$. This implies $u \leq w^{t}$ in $B_{r}(y)$ for all $t \in\left[0, t_{0}\right]$. For otherwise, since $u \leq v=w^{0}$, there would exist $t \in\left(0, t_{0}\right]$ such that $u \leq w^{t}$ in $B_{r}(y)$ and $u=w^{t}$ at some interior point, contradicting (4.5) and the maximum principle.

Consequently,

$$
(v-u)(x) \geq t_{0}(h-v)(x) \geq t_{0} \theta_{0} \operatorname{dist}_{\mathbb{S}^{n}}\left(x, \partial B_{r}(y)\right) \quad \text { for } x \in B_{r}(y) .
$$

In particular,

$$
(u-\underline{u})(y) \geq t_{0} \theta_{0} r=\frac{t_{0} \theta_{0}}{3} \operatorname{dist}_{\mathbb{S}^{n}}(y, \partial \Omega),
$$

completing the proof. 
4.1. Proof of Theorem 4.1. Write $\eta \equiv v-u$ and set

$$
M=\max _{x \in \bar{\Omega}} \max _{|\xi|=1, \xi \in T_{x} \mathbb{S}^{n}} \eta\left(u+\nabla_{\xi}^{2} u\right) \exp \frac{a}{2}\left(u^{2}+|\nabla u|^{2}\right),
$$

where $a>0$ is a constant to be determined later. We shall first derive a bound for $M$. It is similar to the proof of the global estimate for second derivatives in Section 2, but here we need some more precise inequalities.

Since $\eta=0$ on $\partial \Omega, M$ is achieved at some interior point $x_{0} \in \Omega$ and for some unit vector $\xi \in T_{x_{0}} \mathbb{S}^{n}$. We may choose a smooth orthonormal local frame $e_{1}, \ldots, e_{n}$ about $x_{0}$ such that $e_{1}\left(x_{0}\right)=\xi$ and $\left\{\nabla_{i j} u\left(x_{0}\right)\right\}$ is diagonal. Set

$$
\lambda_{i}=\left(u+\nabla_{i i} u\right)\left(x_{0}\right)>0 \text { for } i=1, \ldots, n .
$$

The function $\log \eta+\log \left(u+\nabla_{11} u\right)+\frac{a}{2}\left(u^{2}+|\nabla u|^{2}\right)$ (defined near $\left.x_{0}\right)$ then attains a maximum at $x_{0}$ where

$$
\frac{\eta_{i}}{\eta}+\frac{\nabla_{i} u+\nabla_{i 11} u}{\lambda_{1}}+a \lambda_{i} \nabla_{i} u=0 \quad \text { for all } i
$$

and

$$
\begin{aligned}
0 \geq \frac{\nabla_{i i} \eta}{\eta}-\left(\frac{\nabla_{i} \eta}{\eta}\right)^{2}+\frac{\nabla_{i i} u+\nabla_{i i 11} u}{\lambda_{1}}-\frac{\left(\nabla_{i} u+\nabla_{i 11} u\right)^{2}}{\lambda_{1}^{2}} & \\
& +a \lambda_{i} \nabla_{i i} u+a \sum_{j} \nabla_{j} u\left(\nabla_{i i j} u+\delta_{i j} \nabla_{i} u\right), \text { for all } i .
\end{aligned}
$$

From (4.7) we have for $i \geq 2$,

$$
\left(\frac{\nabla_{i} \eta}{\eta}\right)^{2}=\frac{\left(\nabla_{i} u+\nabla_{i 11} u\right)^{2}}{\lambda_{1}^{2}}-\frac{2 a \lambda_{i} \nabla_{i} u \nabla_{i} \eta}{\eta}-\left(a \lambda_{i} \nabla_{i} u\right)^{2}
$$

We need make use the following formulas which are obtained by differentiating (1.1),

$$
\sum_{i} \frac{\nabla_{j} u+\nabla_{j i i} u}{\lambda_{i}}=n \nabla_{j}(\log \psi) \quad \text { for all } j
$$


$(4.10)$

$$
\sum_{i} \frac{\nabla_{11} u+\nabla_{11 i i} u}{\lambda_{i}}-\sum_{i, j} \frac{\left(\nabla_{1} u \delta_{i j}+\nabla_{1 i j} u\right)^{2}}{\lambda_{i} \lambda_{j}}=n \nabla_{11}(\log \psi) .
$$

From (1.13) we find

$$
\sum_{i, j} \frac{\left(\nabla_{1} u \delta_{i j}+\nabla_{1 i j} u\right)^{2}}{\lambda_{i} \lambda_{j}}-\frac{\left(\nabla_{1} u+\nabla_{111} u\right)^{2}}{\lambda_{1}^{2}}-2 \sum_{i>1} \frac{\left(\nabla_{i} u+\nabla_{i 11} u\right)^{2}}{\lambda_{1} \lambda_{i}} \geq 0 .
$$

Multiplying (4.8) by $\lambda_{1} \lambda_{i}^{-1}$, by (4.10) and (1.14), we see that

$$
\begin{aligned}
0 \geq n & \nabla_{11}(\log \psi)+\sum_{i} \frac{1}{\lambda_{i}}\left(\nabla_{11} u-\nabla_{i i} u\right) \\
& +\frac{\lambda_{1}}{\eta} \sum_{i} \frac{\nabla_{i i} \eta}{\lambda_{i}}-\left(\frac{\nabla_{1} \eta}{\eta}\right)^{2}-\frac{C a \lambda_{1}}{\eta} \\
& +a \lambda_{1} \sum_{i}\left(\lambda_{i}-u\right)+a \lambda_{1} \sum_{i, j} \frac{1}{\lambda_{i}} \nabla_{j} u\left(\nabla_{i i j} u+\delta_{i j} \nabla_{i} u\right)
\end{aligned}
$$

Setting $f=n \log \psi$, we calculate

$$
\begin{aligned}
\nabla_{11} f & \geq \sum_{j} f_{p_{j}} \nabla_{11 j} u-C\left(1+\lambda_{1}^{2}\right) \\
& \geq-a \lambda_{1} \sum_{j} f_{p_{j}} \lambda_{j} \nabla_{j} u-C\left(1+\lambda_{1} \eta^{-1}+\lambda_{1}^{2}\right)
\end{aligned}
$$

by (4.7). From (4.9) and (1.13),

$$
\begin{aligned}
\sum_{i, j} \frac{1}{\lambda_{i}} \nabla_{j} u\left(\nabla_{i i j} u+\delta_{i j} \nabla_{i} u\right) & =\sum_{j} \nabla_{j} u \nabla_{j} f \\
& \geq \sum_{j} f_{p_{j}} \lambda_{j} \nabla_{j} u-C .
\end{aligned}
$$

Hence

$$
\nabla_{11} f+a \lambda_{1} \sum_{i, j} \frac{1}{\lambda_{i}} \nabla_{j} u\left(\nabla_{i i j} u+\delta_{i j} \nabla_{i} u\right) \geq-C-C \lambda_{1}\left(a+\eta^{-1}+\lambda_{1}\right) .
$$

Now multiply (4.11) by $\eta^{2}$, and note that

$$
\nabla_{i i} \eta=\left(v+\nabla_{i i} v\right)-(v-u)-\left(u+\nabla_{i i} u\right) \geq-\eta-\lambda_{i},
$$


since $\left\{v+\nabla_{i j} v\right\}>0$. We find

$$
0 \geq(a-C) M^{2}-C(1+a) M-C,
$$

from which follows a bound for $M$ when $a \geq 1+C$. Finally, at any point $x \in \Omega$, we have

$$
\max _{|\xi|=1, \xi \in T_{x} \mathbb{S}^{n}}\left(u+\nabla_{\xi}^{2} u\right)(x) \leq \frac{M}{\eta(x)} \exp \frac{-a}{2}\left(u^{2}+|\nabla u|^{2}\right),
$$

and therefore, by Lemma 4.2,

$$
\max _{|\xi|=1, \xi \in T_{x} \mathbb{S}^{n}}\left(u+\nabla_{\xi}^{2} u\right)(x) \leq \frac{C}{\operatorname{dist}_{\mathbb{S}^{n}}(x, \partial \Omega)},
$$

which implies (4.2).

4.2. Proof of Theorem 1.2. From (2.4) we see that

$$
\psi_{0} \equiv \inf _{x \in \bar{\Omega}} \inf _{u \in \mathcal{A}} \psi(x, u(x), \nabla u(x))>0 .
$$

For each integer $k \geq 1$ set

$$
\psi^{k}(x, z, p) \equiv \psi(x, z, p)-\frac{1}{2 k} \psi_{0}
$$

and consider the Dirichlet problem for

$$
\left(g^{-1} \operatorname{det}\left(u g_{i j}+\nabla_{i j} u\right)\right)^{1 / n}=\psi^{k}(x, u, \nabla u) \quad \text { in } \bar{\Omega},
$$

with boundary condition (1.2). We note that $\underline{u}$ is a strict subsolution of (4.15). Thus it follows from Theorem 1.1 that (4.15)-(1.2) admits a solution $u^{k} \in \mathcal{A}$ for each $k \geq 1$. By $(2.4)$ we have a uniform $C^{1}$ bound

$$
\left\|u^{k}\right\|_{C^{1}(\bar{\Omega})} \leq C_{0} \quad \text { independent of } k .
$$

We do not have global a priori estimates independent of $k$ for second derivatives. But we can apply Theorem 4.1 to $u^{k}$ (with $v=u^{1}$ ) to obtain, for all $k \geq 2$,

$$
\left|\nabla^{2} u^{k}(x)\right| \leq \frac{C}{\operatorname{dist}_{\mathbb{S}^{n}}(x, \partial \Omega)}, \quad \text { for } x \in \Omega .
$$

Here $C$ is a uniform constant independent of $k$. It follows from the $C^{2, \alpha}$ interior estimate of L. C. Evans [4] that for any subdomain $\Omega^{\prime} \Subset \Omega$,

$$
\mid \nabla^{2} u^{k} \|_{C^{\alpha}\left(\bar{\Omega}^{\prime}\right)} \leq C, \quad \text { uniformly for } k \geq 2 .
$$


Finally, we conclude from (4.16), (4.18) and the standard regularity theory that $u^{k}$ has a subsequence which converges to a solution $u \in C^{\infty}(\Omega) \cap C^{0,1}(\bar{\Omega})$ of (1.1)-(1.2).

To conclude this section, we remark that Theorem 1.3 may be similarly proved. Moreover, it holds for the more general Monge-Ampère equations

$$
\left(\left(\operatorname{det}\left(u_{i j}\right)\right)^{1 / n}=\psi(x, u, D u)\right.
$$

with $\psi>0$ being a convex function of the gradient $D u$. In place of (4.15) we may consider

$$
\left(\left(\operatorname{det}\left(u_{i j}\right)\right)^{1 / n}=\psi(x, u, D u)-\frac{\psi_{0}}{2 k}\right.
$$

(where $\psi_{0}$ is similarly defined as in (4.13)) and apply Theorem 0.3 of [5] to obtain a strictly locally convex solution $u^{k} \in C^{\infty}(\bar{\Omega})$ with $u^{k} \geq \underline{u}$ and $\left.u^{k}\right|_{\partial \Omega}=\varphi$ for each $k \geq 1$. We observe that (4.16) still holds, while we can modify the proof of Trudinger and Urbas [10] of the interior estimate for the second derivatives to obtain in place of $(4.17)$

$$
\left|D^{2} u^{k}(x)\right| \leq \frac{C}{\operatorname{dist}_{\mathbb{R}^{n}}(x, \partial \Omega)}, \quad \text { for } x \in \Omega .
$$

Now the Evans Theorem applies to complete the proof of Theorem 1.3 as before.

4.3. Acknowledgment. I wish to thank Professor Joel Spruck, my thesis advisor, for his advice, encouragement and many useful conversations.

\section{REFERENCES}

[1] L. A. Caffareldi, L. Nirenberg and J. Spruck, The Dirichlet problem for nonlinear second-order elliptic equations I. Monge-Ampère equations, Comm. Pure Applied Math., 37 (1984), 369-402.

[2] L. A. Caffarelli, J. J. Kohn, L. Nirenberg and J. Spruck, The Dirichlet problem for nonlinear second-order elliptic equations II. Complex MongeAmpère and uniformly elliptic equations, Comm. Pure Applied Math., 38 (1985), 209-252.

[3] S. Y. Cheng, and S. T. YAu, On the regularity of the solution of the ndimensional Minkowski problem, Comm. Pure Applied Math., 29 (1976), $495-516$.

[4] L. C. Evans, Classical solutions of fully nonlinear, convex, second order elliptic equations, Comm. Pure Applied Math., 35 (1982), 333-363.

[5] B. Guan and J. Spruck, Boundary value problem on $S^{n}$ for surfaces of constant Gauss curvature, Annals of Math., 138 (1993), 601-624.

[6] D. Hoffman, H. Rosenberg and J. Spruck, Boundary value problems for surfaces of constant Gauss curvature, Comm. Pure Applied Math., 45 (1992), 1051-1062. 
[7] N. V. KRylov, Boundedly nonhomogeneous elliptic and parabolic equations in a domain, Izvestia Math. Ser., 47 (1983), 75-108.

[8] H. Rosenberg, Hypersurfaces of constant curvature in space forms, Bull. Sciences Mathematiques, 117 (1993), 211-239.

[9] N. S. Trudinger, On the Dirichlet problem for Hessian equations, preprint.

[10] N. S. Trudinger and J. I. E. Urbas, On second derivative estimates for equations of Monge-Ampère type, Bull. Austral. Math. Soc., 30 (1984), 321-334.

Department of Mathematics

University of Massachusetts

Amherst, Massachusetts 01003

E-mail: guan@gang.umass.edu

Received: September 11, 1994. 